\title{
IMPLICAÇÕES DO VAGINISMO NO COTIDIANO DAS MULHERES
}

\author{
Isabelle Siqueira Lima,' Maria Letícia Pereira de Sousa, ${ }^{2}$ \\ Melissa de Queiroz Carvalho, ${ }^{3}$ Sandra Rebouças Macedo ${ }^{4}$
}

\author{
IMPLICATIONS OF VAGINISMUS IN WOMEN'S DAILY LIVES
}

\section{IMPLICACIONES DEL VAGINISMO EN TODOS LOS DÍAS DE LA MUJER}

\begin{abstract}
Resumo: A taxa de incidência do transtorno da dor sexual feminina/vaginismo varia de $11,7 \%$ a $42 \%$ entre mulheres que apresentam disfunção sexual. Objetivo: descrever implicações do vaginismo no cotidiano das mulheres. Metodologia: estudo de campo, descritivo, transversal e quantitativo, realizado no Grupo de Apoio às Mulheres com Vaginismo, no período de dezembro de 2016 a março de 2017, composto por 5 I mulheres diagnosticadas com vaginismo primário. Foram enviados questionários para serem autoaplicados, contendo aspectos biopsicossociais e da função sexual. Resultados: as participantes $(n=5 \mathrm{I})$ eram predominantemente da faixa etária de 29 a 39 anos (5I\%), evangélicas (29\%) e de religião não específica (29\%), nível superior completo (35\%), casadas/em união estável (55\%). A maioria associou o vaginismo à educação rígida. Em relação à abordagem profissional, $68 \%$ estavam sendo acompanhadas por ginecologistas, $57 \%$ por fisioterapeutas pélvicos, $35 \%$ estavam em fase de diagnóstico e $37 \%$ em tratamento. $O$ intervalo entre as primeiras queixas e o diagnóstico foi de até um ano (45\%). A maioria (53\%) encontrava-se também insatisfeita com o intervalo entre tratamento e cura. $O$ vaginismo foi associado à baixa autoestima em $47 \%$, e $70 \%$ relataram o incentivo do parceiro ao tratamento. A média do total de escore do Female Sexual Function Index foi de 21 ( \pm 8). Conclusão: as mulheres deste estudo apresentaram baixa função sexual, referiram que o vaginismo afeta sua autoestima e relacionaram a disfunção à educação rígida. Também foi identificada a necessidade de uma abordagem profissional mais efetiva, para reduzir o tempo entre as primeiras queixas e a cura.
\end{abstract}

Palavras-chave: Vaginismo. Fisioterapia. Autoestima.

Abstract: The incidence rate of female sexual pain/vaginismus $11.7 \%$ to $42 \%$ among women who have sexual dysfunction. Objective: to describe the implications of vaginismus in the daily life of women. Methodology: a descriptive, cross-sectional and quantitative field study was carried out in the Support Group for Women with Vaginismus, from December 2016 to March 2017. It consisted of 51 women diagnosed with primary vaginismus. Questionnaires were sent to be self-administered, containing biopsychosocial aspects and sexual function. Results: participants $(n=5 \mathrm{I})$ were predominantly from the age of 29 to 39 years (51\%), evangelical (29\%) and with non-specific religion (29\%), complete upper level (35\%), married/in stable union (55\%). Most associated vaginismus with rigid upbringing. Regarding the professional approach, $68 \%$ were being followed by gynecologists, $57 \%$ by pelvic physiotherapists, $35 \%$ were in the diagnostic phase and $37 \%$ in treatment. The interval between the first complaints and the diagnosis was up to one year (45\%). The majority (53\%) were also dissatisfied with the interval between treatment and cure. Vaginismus was associated with low self-esteem in $47 \%$ and $70 \%$ reported the partner's incentive to treatment. The mean of the total Female Sexual Function Index score was $2 \mathrm{I}( \pm 8)$. Conclusion: the women in this study had low sexual function,

\footnotetext{
Pós-graduada em Fisioterapia Pélvica - Uroginecologia Funcional (Faculdade Inspirar). Egressa do Centro Universitário Christus - Campus Parque Ecológico, Fortaleza, Ceará, Brasil. E-mail: isabellepubli@yahoo.com.br.

2 Graduada em Fisioterapia pelo Centro Universitário Christus. Egressa do Centro Universitário Christus- Campus Parque Ecológico, Fortaleza, Ceará, Brasil. E-mail: mleticia_pereira@yahoo.com.br.

3 Pós-graduanda em Terapia Intensiva pela Escola de Saúde Pública e em Fisioterapia Cardiorrespiratória pela NacionalFisio. Egressa do Centro Universitário Christus - Campus Parque Ecológico, Fortaleza, Ceará, Brasil. E-mail: melissadequeirozcarvalho@gmail.com.

4 Doutora em Ciências - Departamento de Ginecologia - Universidade Federal de São Paulo - Unifesp (20I8). ${ }^{2}$ Docente, Centro Universitário Christus - Campus Parque Ecológico, Fortaleza, Ceará, Brasil. E-mail: sandrarmacedo@yahoo.com.br.
} 
reported that vaginismus affects their self-esteem and related dysfunction to rigid education. The need for a more effective professional approach was also identified to reduce the time between first complaints and cure.

Keywords: Vaginismus. Physiotherapy. Self esteem.

Resumen: La tasa de incidencia del trastorno de dolor sexual femenino / vaginismo varía de I I.7\% a 42\% entre las mujeres que tienen disfunción sexual. Objetivo: describir las implicaciones del vaginismo en la vida cotidiana de las mujeres. Metodología: estudio de campo, descriptivo, transversal y cuantitativo, realizado en el Grupo de Apoyo a Mujeres con Vaginismo, de diciembre de 2016 a marzo de 2017, compuesto por 51 mujeres diagnosticadas con vaginismo primario. Se enviaron cuestionarios para autoadministrarse, que contienen aspectos de función biopsicosocial y sexual. Resultados: los participantes $(n=5$ I) eran predominantemente del grupo de edad de 29 a 39 años (5I \%), evangélicos (29\%) y religión no específica (29\%), educación superior completa (35\%), casados / a unión estable (55\%). La mayoría de los vaginismos asociados con la educación estricta. En cuanto al enfoque profesional, el $68 \%$ estaban acompañados por ginecólogos, el $57 \%$ por fisioterapeutas pélvicos, el 35\% estaban en la fase de diagnóstico y el 37\% estaban en tratamiento. El intervalo entre las primeras quejas y el diagnóstico fue de hasta un año (45\%). La mayoría (53\%) tampoco estaba satisfecha con el intervalo entre el tratamiento y la cura. El vaginismo se asoció con baja autoestima en el $47 \%$, y el $70 \%$ informó el incentivo de la pareja para el tratamiento. El promedio de la puntuación total del Índice de Función Sexual Femenina fue de 2 I ( \pm 8). Conclusión: las mujeres en este estudio tenían baja función sexual, informaron que el vaginismo afecta su autoestima y relacionó la disfunción con una educación estricta. También se identificó la necesidad de un enfoque profesional más efectivo, para reducir el tiempo entre las primeras quejas y la cura.

Palabras clave: Vaginismo. Fisioterapia. Autoestima.

\section{Introdução}

O Transtorno da Dor Sexual Feminina (TDSF) passou a ter nova nomenclatura a partir de 2013 pelo (DSM 5), no qual o vaginismo e a dispareunia foram condensados em uma única disfunção, passando a ser chamada, a partir daí, de transtorno de dor genitopélvica/ penetração (BRASIL; ABDO, 20I6). Para fins didáticos, neste estudo, será utilizado o termo vaginismo (que está dentro do conceito de TDSF), pois este estudo trata desta questão específica.

Moreira (2013) afirma que o vaginismo se apresenta como uma contração recorrente ou persistente; quando se tenta a penetração vaginal com o pênis, dedo, tampão ou espéculo. Pode também ocorrer perante a antecipação da introdução vaginal. Beutel, Stöbel-Richter e Brähler (2008) Consideram o vaginismo decorrente de traumas na infância por violência sexual, como abuso sexual e estupro, ocasionando impacto negativo na função sexual (FS). Contudo, o histórico de educação sexual rígida, seja moral, religiosa ou ambas, é o mais comum (AVEIRO; GARCIA; DRIUSSO, 2009).

Segundo Smith e outros (2007), a mulher tem recorrido aos cuidados médicos, com mais frequência, em busca de solução para os problemas que interferem na sua Qualidade de Vida (QV), em especial aqueles relacionados com sua função sexual.

Porém, grande parcela das mulheres ainda resiste à ajuda médica, por vergonha, por frustração ou por falhas de tentativas de tratamento subprofissionalizado (LARA et al., 2008).

Atualmente, é cada vez mais reconhecida a importância da saúde sexual para a longevidade das relações afetivas e como parte da saúde global e bem-estar do indivíduo (LARA et al., 2008).

Sabe-se que o vaginismo afeta a QV, comprometendo a vida sexual ou conjugal, condicionando o bem-estar desses casais ao possível declínio gradativo da autoestima da parceira, o que interfere diretamente em suas vidas social e pessoal (TRINDADE; FERREIRA, 2008).

Já nas mulheres, o ato sexual desperta um determinante maior que o próprio impulso sexual, também ligado à moral, religião, lealdade, decência e segurança (SERRA, 2009). Contudo, as mulheres com vaginismo geralmente apresentam desejo, excitação e orgasmo através de outras formas de relação em que não ocorra à penetração, bem como também lubrificação vaginal e orgasmos, mas não conseguem realizar o coito (ANTONIOLI; SIMÕES, 2010).

Segundo Moreira (20I3) relata em seu estudo, que há, geralmente, o desconhecimento do tema por parte da paciente e do cônjuge. Muitas vezes, o casal já passou por vários profissionais que também desconhecem esse problema e lhes deram informações inadequadas. Por esse motivo, considera que a primeira providência é informar sobre a DS e assegurar que há tratamentos disponíveis que, em geral, não são os propostos por leigos ou por profissionais desavisados. 
O mesmo autor descreve que muitos profissionais desconhecem o vaginismo, o que faz com que as pacientes sejam submetidas a uma sucessão de profissionais, muitas vezes com tratamentos inadequados e com efeitos adversos. Moreira (2013, p. 337) acrescenta que:

muitas mulheres relatam ser tratadas como neuróticas ou difíceis e acusadas de não colaborarem com o exame médico. Às vezes, referem que o exame ginecológico é como um estupro, já que a questão básica dessa disfunção sexual é a incapacidade em permitir a penetração vaginal, seja através do ato sexual, do exame ginecológico ou em alguma outra situação.

Diante do exposto, percebe-se que o médico especialista em Saúde da Mulher não tem abordado objetivamente aspectos da sexualidade feminina de forma sensível, gerando, com isso, um diagnóstico tardio e abordagem inadequada do vaginismo. Tal fato também foi observado através da experiência clínica de uma das pesquisadoras, bem como o quanto é difícil para essas mulheres verbalizarem suas queixas, dificuldades e intimidade com um profissional de saúde.

Tozo e outros (2007) concluem, em seu estudo, que, os profissionais da área da saúde, em geral, não estão adequadamente preparados para abordar as queixas sexuais dos pacientes. Ainda segundo os autores, isto pode estar relacionado a pouco conhecimento sobre o assunto. Foi observado também, no estudo, a carência de disciplinas que abordem exclusivamente sobre sexualidade humana na grade curricular dos cursos de graduação.

Justifica-se o desenvolvimento deste estudo, pela relevância do tema na QV das mulheres, no sentido de melhor compreender o assunto envolvendo aspectos das mulheres e de seus parceiros.

O estudo teve o objetivo de conhecer as implicações do vaginismo no cotidiano das mulheres; traçar seu perfil sociodemográfico; identificar intervalos entre o diagnóstico, o - tratamento e a - cura do vaginismo; conhecer a satisfação das mulheres com vaginismo em relação ao tratamento; e investigar aspectos relacionados à participação do parceiro.

\section{Metodologia}

A pesquisa tratou-se de um estudo de campo, do tipo descritivo, transversal, de natureza quantitativa. Foi realizada seguindo os preceitos da resolução $n^{\circ} 466 / 12$ do Conselho Nacional de Saúde, Comissão Nacional de Ética em Pesquisa do Ministério da Saúde (20I2), que dispõe sobre pesquisas envolvendo seres humanos.

O estudo foi aprovado pelo Comitê de Ética (CEP) do Centro Universitário Christus (Unichristus) com parecer I.729.103 e CAAE 587936।6.4.0000.5049. Ocorreu no período de dezembro de 2016 a março de
2017, em um grupo fechado denominado "Grupo de apoio a mulheres com vaginismo," localizado em uma grande rede social, onde é discutido o tema e esclarecidas dúvidas, bem como dadas sugestões sobre o assunto por parte das integrantes do grupo.

A população geral do grupo onde foram coletados os dados aproximava-se de 1500 mulheres, divididas entre profissionais que abordam o vaginismo e mulheres com queixa sexuais, dentre elas, as diagnosticadas com vaginismo, outras que apresentavam sintomas comuns e outras que já tinham obtido a cura. Dessa forma, foram incluídas na pesquisa mulheres maiores de 18 anos, diagnosticadas com vaginismo primário (quando a mulher nunca experimentou relação sexual com penetração indolor), estando em uma das fases, como diagnóstico, tratamento ou cura. Não foram incluídas na pesquisa as profissionais participantes do grupo e, mulheres diagnosticadas com vaginismo secundário, ou seja, quando ela já experienciou anteriormente relação sexual não dolorosa com penetração.

Os instrumentos de avaliação, elaborados pelas pesquisadoras, foram baseados em questionários de condição geral e específica, relacionados à saúde e função sexual e conduzido pela ferramenta Formulário Google, ${ }^{5}$ contendo questões de múltipla escolha abordando o perfil sociodemográfico das mulheres com vaginismo, suas implicações no cotidiano, os intervalos entre diagnóstico, tratamento e cura do vaginismo e a satisfação dessas mulheres em relação ao tratamento.

Inicialmente, todas as participantes do grupo foram convidadas a participar do estudo. Para aquelas que aceitaram, foram enviados três : o primeiro, explicativo e convidativo, abordava a pergunta "Você está no grupo por ter vivenciado ou estar vivenciando o vaginismo primário (nunca ter tido penetração vaginal)?", como também o Termo de Consentimento Livre e Esclarecido (TCLE) em anexo, no qual o sujeito da pesquisa é esclarecido sobre o objetivo do estudo, a identificação dos pesquisadores e a informação de confidencialidade assegurada, além de esclarecer que, ao responder o questionário estará concordando concomitantemente com sua participação. O segundo baseava-se em um questionário voltado para aspectos gerais, já citados no parágrafo anterior; e o terceiro enviado apresentava o Female Sexual Function Index (FSFI). Trata-se de um questionário breve, com 19 questões, originalmente da língua inglesa, autoaplicativo, específico e multidimensional, que avalia: desejo sexual, excitação, lubrificação vaginal, orgasmo, satisfação e dor.

\footnotetext{
Disponível através dos links: <https://docs.google.com/forms/d/IHP I 3lmPFygT I wuJvHc8qPmFGxd|2GgOq4QidU9-WZuc/viewform?chromeless $=$ I \&edit_requested =true $>$ e $<$ https://docs.google.com/ forms/d/IKJ|KcGBgeAItBwaase8DblHmolDg2]Zgba_oFu3qucM/viewform?chromeless $=\mid$ \&edit_requested $=$ true $>$
} 
Em 2008, o FSFI foi traduzido para a língua portuguesa, adaptado culturalmente e validado para avaliar o índice da função sexual em brasileiras (MAGNO; FONTESPEREIRA; NUNES, 20I I).

Aquelas que responderam "sim" à pergunta do primeiro passaram a fazer parte do estudo, totalizando $5 \mathrm{I}$ participantes, onde a maioria era da região Sul e Sudeste. Em relação à possibilidade de vieses, pode haver em caso de alguma participante que não soube responder às perguntas ou omitiu alguma resposta. A pesquisadora esteve à disposição para esclarecer qualquer dúvida das participantes por. Os dados colhidos foram utilizados somente para esse fim, sendo preservadas suas identidades.

Os dados coletados foram armazenados em um banco de dados com o auxílio do Microsoft Office Excel versão 2010 e posteriormente analisados pelo estatístico Statistical Package for the Social Science (SPSS) versão
20.0. Foi realizada estatística descritiva e utilizado recurso da frequência para descrever o perfil sociodemográfico, destacar suas implicações no cotidiano, conhecer a satisfação das mulheres com vaginismo em relação ao tratamento, investigar a participação do parceiro no tratamento; e ainda fazer comparações entre médias utilizando o teste paramétrico T-Student em pares, para identificar intervalos entre diagnóstico, - tratamento e - cura do vaginismo. Os resultados foram expostos em quadros para melhor compreensão e embasamento com a literatura.

\section{Resultados}

A maioria das participantes era jovem, com predomínio do nível superior completo, casadas/em união estável. Quarenta e sete mulheres não se submeteram a nenhum tipo de parto $(92,2 \%)$ (Tabela I).

Tabela I - Características sociodemográficas das participantes

\begin{tabular}{|c|c|}
\hline & $\mathbf{N}(\%)$ \\
\hline \multicolumn{2}{|l|}{ Idade } \\
\hline 18 a 28 anos & $23(45,1)$ \\
\hline 29 a 39 anos & $26(5 I)$ \\
\hline 40 a 50 anos & I (2) \\
\hline 51 e mais & I (2) \\
\hline \multicolumn{2}{|l|}{ Região } \\
\hline Centro-Oeste & $4(7,8)$ \\
\hline Nordeste & $4(7,8)$ \\
\hline Norte & $4(7,8)$ \\
\hline Sul & $17(33,3)$ \\
\hline Sudeste & $22(43,1)$ \\
\hline \multicolumn{2}{|l|}{ Religião } \\
\hline Católica & II $(2 \mid, 6)$ \\
\hline Evangélica & $15(29,4)$ \\
\hline Protestante & $4(7,8)$ \\
\hline Testemunho de Jeová & $2(3,9)$ \\
\hline Espírita & $4(7,8)$ \\
\hline Religião não específica e/ou outras opções & I5 $(29,4)$ \\
\hline \multicolumn{2}{|l|}{ Escolaridade } \\
\hline Ensino Médio Completo & II $(2 \mid, 6)$ \\
\hline Ensino Superior Incompleto & $13(25,5)$ \\
\hline Superior Completo & $18(35,3)$ \\
\hline Pós-Graduação & $9(17,6)$ \\
\hline \multicolumn{2}{|l|}{ Estado Civil } \\
\hline Solteira & $22(43,1)$ \\
\hline
\end{tabular}




\begin{tabular}{l|l} 
& N $(\%)$ \\
\hline Casada/União Estável & $28(54,9)$ \\
\hline Separada/Divorciada & $\mathrm{I}(2)$ \\
\hline
\end{tabular}

$\mathrm{Na}$ abordagem da Disfunção Sexual Feminina (DSF), é fundamental conhecer aspectos da sexualidade para melhor compreender as implicações dos sintomas na QV da paciente. No estudo, boa parte das participantes relatou estar na fase de tratamento do vaginismo, seguida da fase de diagnóstico. Apresentavam parceiros fixos nos últimos 6 meses, como também tiveram penetração total no mesmo período (Tabela 2).

Tabela 2 - Aspectos da sexualidade

\begin{tabular}{l|l} 
Fases do vaginismo & $\mathbf{N}(\%)$ \\
\hline Diagnóstico & $18(35,3)$ \\
\hline Tratamento & $19(37,3)$ \\
\hline Parceiros fixos nos últimos 6 meses & \\
\hline Sim & $44(86,3)$ \\
\hline Não & $7(13,7)$ \\
\hline Penetração total nos últimos 6 meses & \\
\hline Sim, parcialmente & $16(31,4)$ \\
\hline Sim, totalmente & $20(39,2)$ \\
\hline Não & $15(29,4)$ \\
\hline
\end{tabular}

A maioria buscou, respectivamente, a abordagem profissional de ginecologistas e fisioterapeutas pélvicos, como ilustrado na Tabela 3.

Tabela 3 - Abordagem profissional

\begin{tabular}{l|l}
\hline & N $(\%)$ \\
\hline Psicóloga & $21(41,2)$ \\
\hline Sexóloga & $6(11,8)$ \\
\hline Psicóloga/Sexóloga & $3(5,9)$ \\
\hline Ginecologista & $35(68,6)$ \\
\hline Fisioterapeuta & $29(56,9)$ \\
\hline Outros (dança, yoga etc....) & $8(15,7)$ \\
\hline
\end{tabular}

Muitas mulheres $(n=28)$, deste estudo, relataram apresentar satisfação com a abordagem profissional (55\%). Contudo, 27 delas sentem-se insatisfeitas com o intervalo de tempo entre tratamento e cura (53\%), bem como tiveram empatia com o profissional e ainda relataram a satisfação com as técnicas adotadas.

A abordagem profissional considerada como a melhor para a cura do vaginismo foi a do fisioterapeuta $(n=24 ; 47,1 \%)$. Segundo as avaliadas, o que pode ser melhorado na abordagem profissional é o encaminhamento para profissionais especialistas (68\%).
A educação rígida dos pais foi associada ao vaginismo por número significativo de mulheres $(n=27 ; 53 \%)$.

Quanto aos aspectos do parceiro, as mulheres afirmaram que muitos deles $(n=45 ; 88 \%)$ mostraram-se compreensíveis em relação aos sintomas do vaginismo e $70 \%(n=36)$ relataram ser incentivadas pelos mesmos a realizar o tratamento.

Em relação às atividades diárias (Tabela 4), as mulheres relataram que $\circ$ vaginismo não interfere nas suas atividades sociais $(n=20 ; 39,2 \%)$ nem nas suas atividades físicas $(n=36 ; 70,6 \%)$. Porém, interfere bastante na sua imagem corporal e autoestima $(n=18 ; 35,3 \%)$. 
Quando indagadas sobre o uso de medicamentos ansiolíticos ou antidepressivos por causa do vaginismo, um alto percentual relatou a não utilização de fármacos $(n=43 ; 84,3 \%)$.

Em resposta às questões referentes à $\mathrm{QV}$, nos aspectos emocionais, na variável que questiona se as avaliadas referem sentir ansiedade, teve como a principal resposta foi "maior parte do tempo". Apesar deste fato, afirmaram sentir-se uma pessoa feliz "em uma boa parte do tempo" (Tabela 5).

Tabela 4 - Interferência nas atividades diárias

\begin{tabular}{l|l|l|l|l|l}
\hline Perguntas/Respostas & $\begin{array}{l}\text { De forma } \\
\text { nenhuma }\end{array}$ & Ligeiramente & Moderadamente & Bastante & Extremamente \\
\hline & $\mathbf{N} \%$ & $\mathbf{N} \%$ & $\mathbf{N} \%$ & $\mathbf{N} \%$ & $\mathbf{N} \%$ \\
\hline $\begin{array}{l}\text { De que maneira o vaginismo } \\
\text { interferiu ou interfere nas suas } \\
\text { atividades sociais? }\end{array}$ & $\begin{array}{l}\mathrm{N}=20 \\
39,2 \%\end{array}$ & $\begin{array}{l}\mathrm{N}=13 \\
25,5 \%\end{array}$ & $\begin{array}{l}\mathrm{N}=12 \\
23,5 \%\end{array}$ & $\begin{array}{l}\mathrm{N}=4 \\
7,8 \%\end{array}$ & $\begin{array}{l}\mathrm{N}=2 \\
3,9 \%\end{array}$ \\
\hline $\begin{array}{l}\text { De que maneira o vaginismo } \\
\text { interfere ou interferiu nas suas } \\
\text { atividades físicas? }\end{array}$ & $\begin{array}{l}\mathrm{N}=36 \\
70,6 \%\end{array}$ & $\begin{array}{l}\mathrm{N}=5 \\
9,8 \%\end{array}$ & $\begin{array}{l}\mathrm{N}=8 \\
15,7 \%\end{array}$ & $\begin{array}{l}\mathrm{N}=2 \\
3,9 \%\end{array}$ & \\
\hline $\begin{array}{l}\text { De que maneira o vaginismo } \\
\text { interferiu ou interfere na sua } \\
\text { imagem corporal/autoestima? }\end{array}$ & $\begin{array}{l}\mathrm{N}=5 \\
9,8 \%\end{array}$ & $\begin{array}{l}\mathrm{N}=5 \\
9,8 \%\end{array}$ & $\begin{array}{l}\mathrm{N}=12 \\
23,5 \%\end{array}$ & $\begin{array}{l}\mathrm{N}=18 \\
35,3 \%\end{array}$ & $\begin{array}{l}\mathrm{N}=1 \mathrm{I} \\
2 \mathrm{I}, 6 \%\end{array}$ \\
\hline
\end{tabular}

Em relação ao intervalo de tempo, entre as primeiras queixas e o diagnóstico do vaginismo, muitas mulheres $(n=23 ; 45,1 \%)$ destacaram um período de até 12 meses. O mesmo intervalo de tempo foi relatado entre o diagnóstico e o início do tratamento $(\mathrm{n}=$ 20; 39,2\%). Quanto à abordagem do intervalo de tempo entre o tratamento e a cura, $9(17,6 \%)$ destacaram o período de até 12 meses.

Através das análises dos domínios do FSFI, a média da FS foi de 20,9 ( $\pm 8,0)$, evidenciando DS das participantes do estudo. Observou-se que poucas mulheres $(n=12 ; 23,5 \%)$ apresentaram boa função sexual.

Tabela 5 - Qualidade de vida

\begin{tabular}{|c|c|c|c|c|c|c|}
\hline Perguntas/Respostas & Todo tempo & $\begin{array}{l}\text { A maior } \\
\text { parte do } \\
\text { tempo }\end{array}$ & $\begin{array}{l}\text { Uma boa } \\
\text { parte do } \\
\text { tempo }\end{array}$ & $\begin{array}{l}\text { Alguma } \\
\text { parte do } \\
\text { tempo }\end{array}$ & $\begin{array}{l}\text { Uma } \\
\text { pequena } \\
\text { parte do } \\
\text { tempo }\end{array}$ & Nunca \\
\hline & $\mathbf{N} \%$ & $\mathbf{N} \%$ & $\mathbf{N} \%$ & $\mathbf{N} \%$ & $\mathbf{N} \%$ & $\mathbf{N} \%$ \\
\hline $\begin{array}{l}\text { Quanto tempo você tem } \\
\text { se sentido uma pessoa } \\
\text { muito nervosa? }\end{array}$ & $\begin{array}{l}N=6 \\
11,8 \%\end{array}$ & $\begin{array}{l}N=15 \\
29,4 \%\end{array}$ & $\begin{array}{l}N=11 \\
21,6 \%\end{array}$ & $\begin{array}{l}N=7 \\
13,7 \%\end{array}$ & $\begin{array}{l}N=9 \\
17,6 \%\end{array}$ & $\begin{array}{l}N=3 \\
5,9 \%\end{array}$ \\
\hline $\begin{array}{l}\text { Quanto tempo você tem } \\
\text { se sentido tão deprimida } \\
\text { que nada pode animá-la? }\end{array}$ & $\begin{array}{l}N=3 \\
6 \%\end{array}$ & $\begin{array}{l}N=8 \\
15,7 \%\end{array}$ & $\begin{array}{l}N=9 \\
17,6 \%\end{array}$ & $\begin{array}{l}N=7 \\
13,7 \%\end{array}$ & $\begin{array}{l}N=18 \\
35,3 \%\end{array}$ & $\begin{array}{l}N=6 \\
11,8 \%\end{array}$ \\
\hline $\begin{array}{l}\text { Quanto tempo você } \\
\text { tem se sentido calma ou } \\
\text { tranquila? }\end{array}$ & & $\begin{array}{l}N=10 \\
19,6 \%\end{array}$ & $\begin{array}{l}N=8 \\
I 5,7 \%\end{array}$ & $\begin{array}{l}N=12 \\
23,5 \%\end{array}$ & $\begin{array}{l}N=19 \\
37,3 \%\end{array}$ & $\begin{array}{l}N=2 \\
3,9 \%\end{array}$ \\
\hline $\begin{array}{l}\text { Quanto tempo você tem } \\
\text { se sentido desanimada ou } \\
\text { abatida? }\end{array}$ & $\begin{array}{l}N=4 \\
7,8 \%\end{array}$ & $\begin{array}{l}N=9 \\
17,6 \%\end{array}$ & $\begin{array}{l}N=7 \\
13,7 \%\end{array}$ & $\begin{array}{l}N=15 \\
29,4 \%\end{array}$ & $\begin{array}{l}N=11 \\
21,6 \%\end{array}$ & $\begin{array}{l}N=4 \\
7,8 \%\end{array}$ \\
\hline $\begin{array}{l}\text { Quanto tempo você tem } \\
\text { se sentido uma pessoa } \\
\text { feliz? }\end{array}$ & & $\begin{array}{l}N=14 \\
27,5 \%\end{array}$ & $\begin{array}{l}N=15 \\
29,4 \%\end{array}$ & $\begin{array}{l}N=11 \\
21,6 \%\end{array}$ & $\begin{array}{l}N=9 \\
17,6 \%\end{array}$ & $\begin{array}{l}N=2 \\
3,9 \%\end{array}$ \\
\hline
\end{tabular}




\section{Discussão}

Neste estudo, as mulheres mais jovens apresentaram maior prevalência de vaginismo, quando comparado com a literatura. Conforme Lara e outros (2008), as DSF alcançam alta prevalência em qualquer faixa etária. $O$ vaginismo afeta aproximadamente I em 200 mulheres (SCISCIONE, 2019).

Muitas das avaliadas associaram o vaginismo à educação rígida dos pais, corroborando com o estudo de Lara e outros (2008), quando relatam a prevalência da repressão sexual, tanto da família, quanto social e religiosa, como fatores extrínsecos que contribuem para o surgimento do vaginismo, além de outros fatores como medo da relação sexual, experiências prévias negativas, culto à virgindade e abuso sexual.

Neste trabalho, grande parte das participantes apresentaram nível superior completo. Acerca das variáveis sociodemográficas, Clayton (2007) destaca que mesmo os maiores estudos epidemiológicos sobre DSF, tem apresentado achados contraditórios com relação à etnia, escolaridade, nível socioeconômico etc. Ainda que sem unanimidade, associações entre baixo nível de escolaridade e DSF têm sido registradas na literatura (SHAEER; SHAEER; SHAEER, 20I2). O autor ainda afirma, em seu estudo, que mulheres com maior escolaridade conhecem mais sobre o corpo humano, são menos suscetíveis a tabus e têm maior acesso às informações sobre sexo e saúde em geral.

Contrariando o autor acima, dentre outros estudos encontrados na literatura, Cerejo (2006) relata, em seu estudo, a associação do elevado grau de escolaridade com DSF. Complementa afirmando que este fato pode explicar-se pelo maior estresse existente nessas classes de maior estatuto social, contribuindo para esse resultado.

A abordagem profissional considerada como escolha para o tratamento do vaginismo foi a Fisioterapia. Segundo as avaliadas, o que pode ser melhorado na abordagem profissional dessa disfunção é o encaminhamento para profissionais especialistas, capacitados para tanto.

Complementando essa informação, Martinez (2008) apresenta, em seu estudo, que grande parte das mulheres admite que $\circ$ ginecologista representa um papel fundamental no diagnóstico clínico e manejo das suas dificuldades sexuais e que gostaria que os profissionais fossem mais qualificados nesta área, considerando a variável sobre abordagem do profissional ginecologista. Para Ferreira, Souza e Amorim (2007), a dificuldade de se ter um diagnóstico exato torna o vaginismo obscuro e retarda o início do tratamento.

Este fato em questão é abordado por Reissing (2012), quando considera a relevância de uma abordagem de tratamento multidisciplinar para o vaginismo, de acordo com uma conceituação biopsicossocial.

Lucena e Abdo (20I2) concluem que, o tratamento das queixas sexuais necessita de especial atenção, visto que a disfunção, tendo causa multifatorial, contribui para o estado depressivo em algumas pacientes, devendo participar do tratamento uma equipe multiprofissional com abordagens terapêuticas distintas, respeitando a individualidade de cada caso, com a finalidade de contribuir com a saúde mental, a adesão ao tratamento, a satisfação no relacionamento e a $\mathrm{QV}$.

Quando abordadas em relação aos aspectos do parceiro, as mulheres afirmaram que muitos deles mostraram-se compreensíveis em relação aos sintomas do vaginismo e relataram ser incentivadas pelos mesmos a realizar o tratamento. Este tipo de comportamento é reconhecido por Carvalho e outros (2017), quando afirma ser fundamental que os parceiros sejam incluídos em todas as fases do tratamento, seja pela simples convivência ou por mecanismos de retorno positivo. Complementa ainda que, conforme se desenvolve e cresce a sexualidade da paciente, mais seguro, feliz e realizado se apresentará o casal.

Com as participantes deste estudo, não foi verificado estado depressivo, pois as mulheres afirmaram sentir-se felizes em uma "boa parte do tempo", apesar de nervosas "a maior parte do tempo". Quando indagadas sobre o uso de medicamentos ansiolíticos ou antidepressivos por causa do vaginismo, um alto percentual relatou a não utilização de fármacos. Zemishlany e Weizman (2008) relatam que alguns antidepressivos, particularmente os inibidores da recaptação da serotonina, largamente utilizados em todo o mundo, podem causar disfunção sexual.

Complementando o estudo citado acima, Özdel e outros (2012) afirmam que o vaginismo também está relacionado a componentes da saúde mental, não sendo somente uma DS isolada. Enfatizam, também, que a ansiedade e a personalidade perfeccionista são fatores importantes e devem ser avaliados para o sucesso da conclusão do tratamento.

Nobre e Pinto-Gouveia (2008), com intuito de identificar a influência das variáveis cognitivas e emocionais sobre DSF específicas, sustentam a afirmativa dos autores citados acima, identificando o sentimento de medo como preditor relacionado ao vaginismo.

No estudo, as participantes relataram um intervalo de tempo de 12 meses entre as primeiras queixas e o diagnóstico de vaginismo. Como também afirmaram o mesmo intervalo de tempo entre o diagnóstico e o início do tratamento. Moreira (2013) considera que a terapia sexual e o tratamento físico ocorrem, em média, no período de três a seis meses. Porém, acrescenta que há casos cujo tratamento pode ser mais demorado.

Ao se avaliar, no estudo, a mulher com vaginismo, notou-se a diminuição da sua autoestima, tendo como relato que esta DSF interfere bastante neste fator, além de afetar a imagem corporal. Concordando com este estudo, dados semelhantes foram encontrados por Bravo e outros (20I0), acrescentando à essa disfunção submissão, falta de 
informação sexual, medos sexuais e problemas conjugais. Tais fatores desenvolvem uma frustração no casal, já que a mulher não consegue ter a relação sexual.

Sabe-se que o vaginismo afeta a $\mathrm{QV}$, comprometendo a vida sexual ou conjugal, condicionando o bem-estar desses casais ao possível declínio gradativo da autoestima da parceira, o que interfere diretamente em suas vidas social e pessoal (TRINDADE; FERREIRA, 2008).

Tozo e outros (2007) afirmam que a sexualidade é considerada um dos indicadores de $\mathrm{QV}$, influenciando ações e pensamentos, sentimentos e integrações, portanto, a saúde física e mental. Agrega, também, fatores internos, como afetividade, intelecto e emoção; e fatores externos, como religião, área geográfica, sistema econômico, ambiente social e cultural. Deve, portanto, ser entendida como parte integrante da saúde da mulher.

As mulheres do estudo apresentaram baixa função sexual, já que o ponto de coorte para uma boa função sexual é de 26,5, conforme afirma Lima (2010).

\section{Conclusão}

O vaginismo acomete mulheres jovens, com alta escolaridade, casadas/em união estável, afetando sua autoestima, estando relacionado à educação rígida dos pais, gerando grande impacto na função sexual, segundo este estudo.

A abordagem das queixas sexuais requer medidas gerais que abordem o controle de sintomas somáticos, psíquicos, locais e dificuldades com o parceiro, assim como medidas específicas que considerem as queixas individuais.

Também foi identificada a necessidade de uma abordagem profissional mais efetiva, para reduzir o tempo entre as primeiras queixas e a cura. A capacidade diagnóstica dos profissionais de saúde também é uma questão a ser melhorada, sendo necessário incluir em sua formação orientações específicas voltadas para sexualidade no sentido de reconhecer e tratar esse transtorno no mais curto intervalo de tempo possível.

Novos estudos precisam ser realizados para apurar critérios diagnósticos claros e consensos relacionados à abordagem profissional.

Espera-se, com este estudo, despertar maior interesse no tema por parte dos profissionais da saúde, para que possam melhorar sua abordagem, diagnóstico e encaminhamento.

\section{Referências}

ANTONIOLI, R. S.; SIMÕES, D. Abordagem fisioterapêutica nas disfunções sexuais femininas. Revista Neurociências, v. 18, n. 2, p. 267-274, 2010. Disponível em: http://www.revistaneurociencias. com.br/edicoes/ 2010/RNI802 /374\% 20revisao. pdf. Acesso em: 17 maio 2016.
AVEIRO, M. C.; GARCIA, A. P. U.; DRIUSSO, P. Efetividade de intervenções fisioterapêuticas para o vaginismo: uma revisão da literatura. Fisioterapia e Pesquisa., v. 16, n. 3, p. 279-283, 2009.

Disponível em: http://www.revistas.usp.br/fpusp/ article/view/I2147. Acesso em: 17 maio. 2016.

BEUTEL, M. E; STÖBEL-RICHTER, Y.;

BRÄHLER, E. Sexual desire and sexual activity of men and women across their lifespans: results from a representative German community survey. BJU International. v. I0I, n. I, p. 7682, 2008. Disponível em: https://bjui-journals. onlinelibrary.wiley.com/doi/full/I0. I I I //j. I4644I0X.2007.07204.x

BRASIL, A. P. A.; ABDO, C. H. N. Transtornos sexuais dolorosos femininos. Revista Diagnóstico e Tratamento, São Paulo, v. 21, n. 2, p. 89-92, 2016. Disponível em: http://files.bvs.br/upload/S/I4I3-9 979/20I6/v2 In2/a5592.pdf.

BRASIL. Resolução CNS n 466, I 2 de dezembro de 20I2. Aprova diretrizes e normas de pesquisa envolvendo seres humanos. Diário Oficial da União, Brasília, DF, I 3 jun. 2012, n. 12, seção I, p. 59.

BRAVO, C. S. et al. Perfiles e indicadores psicológicos relacionados con la dispareunia y el vaginismo. Estudio cuantitativo. Segunda parte. Salud mental, Montes Urales, v. 33, n. 4, p. 347-357, 20I0. Disponível em: http://www. scielo.org.mx/scielo.php?script =sci_arttext\&pid $=$ SOI $85-33252010000500008$

CARVALHO, J. C. G. R. et al. Terapêutica multimodal do vaginismo: abordagem inovadora por meio de infiltracão de pontos gatilho e radiofrequência pulsada do nervo pudendo. Revista Brasileira de Anestesiologia. v. 67, n. 6, p. 632-636, 2017.Disponível em: https://www.scielo.br/scielo.php?pid=S0034$70942017000600632 \&$ script $=$ sci_ arttext\&tlng $=p t$

CEREJO, A. C. Disfunção sexual feminina: prevalência e factores relacionados. Revista Portuguesa de Medicina Geral e Familiar, [s. I.], v. 22, n. 6, p. 70I-720, nov. 2006. Disponível em: https://www.rpmgf.pt/ojs/index.php/rpmgf/ article/view/I0303 
CLAYTON, A. H. Epidemiology and neurobiology of female sexual dysfunction. The Journal of Sexual Medicine, v. 4, n. 4, p. 260-268, Nov. 2007. Disponível em: https://www.jsm.jsexmed.org/ article/SI 743-6095(I5)3 I 783-5/fulltext

CLAYTON, A. H.; MONTEJO, A. L. Major depressive desorder, antidepressants, and sexual de dysfunction. The Journal of clinical psychiatry, v. 67, n. 6, p. 33-37, 2006. Disponível em: http:// europepmc.org/article/med/I6848675

FERREIRA, A. L. C. G.; SOUZA, A. I.; AMORIM, M. M. R. Prevalência das disfunções sexuais femininas em clínica de planejamento familiar de um hospital escola no Recife, Pernambuco. Revista Brasileira de Saúde Materno-Infantil. v. 7 , n. 2, p. I43-I50, 2007. Disponível em: https:// www.scielo.br/pdf/rbsmi/v7n2/04.pdf

LARA, L. A. S. et al. Abordagem das disfunções sexuais femininas. Revista Brasileira de Ginecologia e Obstetrícia. Rio de Janeiro, v. 30, n. 6, p. 3|2-32|, 2008. Disponível em: https://www. scielo.br/scielo.php?script $=$ sci_arttext\&pid $=$ SOI00-72032008000600008

LIMA, S. M. R. R. et al. Disfunções sexuais femininas: questionários utilizados para avaliação inicial. Arquivos Médicos dos Hospitais e da Faculdade de Ciências Médicas da Santa Casa de São Paulo, São Paulo, v. 55, n. I, p. I-6, 20 I0. Disponível em: http://arquivosmedicos. fcmsantacasasp.edu.br/index.php/AMSCSP/ article/view/303

LUCENA, B. B; ABDO, C. H. N. Considerações sobre a disfunção sexual feminina e a depressão. Diagnóstico e Tratamento, v. 2, n. 17, p. 82-85, 20/2. Disponível em: http://files.bvs.br/ upload/S/ I 4 |3-9979/20 I2/v17n2/a3024.pdf

MAGNO, L. D. P; PEREIRA, A. J. F; NUNES, E. F. C. Avaliação quantitativa da função sexual feminina correlacionada com a contração dos músculos do assoalho pélvico. Revista PanAmazônica de Saúde, Ananindeua, v. 2, n. 4, dez. 20I I. Disponível em: http://scielo.iec.gov.br/pdf/ rpas/v2n4/v2n4a06.pdf

MARTINEZ, L. More education in the diagnosis and management of sexual dysfunction is needed. Fertility and Sterility, v. 89, n. 4, p. I035, Apr. 2008. Disponível em: https://www.fertstert.org/article/ S00I5-0282(08)00475-5/fulltext
MOREIRA, R. L. B. D. Vaginismo. Revista Médica de Minas Gerais. v. 23, n. 3, p. 336-342, 20I3. Disponível em: http://rmmg.org/artigo/ detalhes/2I 8

NOBRE, P. J.; PINTO-GOUVEIA, J. Cognitive and emotional predictors of female sexual dysfunctions: preliminary findings. Journal of Sex and Marital Therapy, v. 34, n. 4, p. 42-325, 2008. Disponível em: https://www.tandfonline.com/doi/ abs/10.1080/00926230802096358

ÖZDEL, K. et al. Factors that might be predictive of completion of vaginismus treatment. Turk Psikiyatri Derg. v. 23, n. 4, p. 53-248, 2012. Disponível em: https://pubmed.ncbi.nlm.nih. gov/23225।25/

REISSING, E. D. Consultation and treatment history and causal attributions in an online sample of women with lifelong and acquired vaginismus. Journal of Sexual Medicine. v. 9, p. 25I-258, 20 I 2. Disponível em: https://www.jsm.jsexmed.org/ article/SI 743-6095(I5)33735-8/fulltext

SCISCIONE, A. C. Diseases and Disorders: Vaginismus. In: FERRI, F. F. Ferri's Clinical Advisor. [S.n.]: Elsevier, 2019. p. I44I-I44I. Disponível em: https://www.clinicalkey. com/service/content/pdf/watermarked/3s2.0-B9780323530422009020.pdf?locale $=$ pt $B R \&$ searchlndex $=$. Acesso em: 25 jun. 2019.

SERRA, M. Qualidade de vida e disfunção sexual: vaginismo. 2009. 123 f. Dissertação (Mestrado em Psicologia) - Pontifícia Universidade Católica de São Paulo, São Paulo, 2009.

SHAEER, O.; SHAEER, K.; SHAEER, E. The Global Online Sexuality Survey (GOSS): female sexual dysfunction among Internet users in the reproductive age group in the Middle East. The Journal of Sexual Medicine, v. 9, n. 2, p. 4I I-424, 2012. Disponível em: https://www.jsm.jsexmed. org/article/SI 743-6095(I5)33873-X/fulltext

SMITH, L. J. et al. Sex after seventy: a pilot study of sexual function in older persons. The Journal of Sexual Medicine, v. 4, n. 5, p. I247-I253, 2007. Disponível em: https://www.sciencedirect.com/ science/article/abs/pii/SI 7436095 I 531643 X. Acesso em: 12 mar. 2020. 
TOZO, I. M. et al. Disfunção sexual feminina: a importância do conhecimento do diagnóstico pelo ginecologista. Arquivos Médicos dos Hospitais e da Faculdade de Ciências Médicas da Santa Casa de São Paulo, São Paulo, v. 52, n. 3, p. 9-94, 2007. Disponível em: http://arquivosmedicos. fcmsantacasasp.edu.br/index.php/AMSCSP/ article/view/447

TRINDADE, W. R.; FERREIRA, M. A. Sexualidade feminina: questões do cotidiano das mulheres.

Texto \& Contexto Enfermagem, Florianópolis, v. 17, n. 3, p. 4I7-426, 2008. Disponível em: http:// www.scielo.br/scielo.php?script $=$ sci_arttext\&pid $=$ SO I 0407072008000300002 Acesso em: 16 maio 2016.

ZEMISHLANY, Z.; WEIZMAN, A. The impact of mental illness on sexual dysfunction. Advances in Psychosomatic Medicine, v. 29, p. 89-106, 2008.

Disponível em: https://www.karger.com/Article/ Abstract// 26626 